\title{
Brain Mapping: From Anatomics to Informatics
}

*Correspondence to:
Sun W,
Tel: +82-2-2286-1404
Fax: +82-2-929-5696
E-mail: woongsun@korea.ac.kr

Received December 20, 2016

Revised December 23, 2016

Accepted December 23, 2016

\author{
Woong Sun* \\ Department of Anatomy, Korea University College of Medicine, Seoul 02841, Korea
}

\begin{abstract}
Neuronal connectivity determines brain function. Therefore, understanding the full map of brain connectivity with functional annotations is one of the most desirable but challenging tasks in science. Current methods to achieve this goal are limited by the resolution of imaging tools and the field of view. Macroscale imaging tools (e.g., magnetic resonance imaging, diffusion tensor images, and positron emission tomography) are suitable for large-volume analysis, and the resolution of these methodologies is being improved by developing hardware and software systems. Microscale tools (e.g., serial electron microscopy and array tomography), on the other hand, are evolving to efficiently stack small volumes to expand the dimension of analysis. The advent of mesoscale tools (e.g., tissue clearing and single plane ilumination microscopy super-resolution imaging) has greatly contributed to filling in the gaps between macroscale and microscale data. To achieve anatomical maps with gene expression and neural connection tags as multimodal information hubs, much work on information analysis and processing is yet required. Once images are obtained, digitized, and cumulated, these large amounts of information should be analyzed with information processing tools. With this in mind, post-imaging processing with the aid of many advanced information processing tools (e.g., artificial intelligence-based image processing) is set to explode in the near future, and with that, anatomic problems will be transformed into informatics problems.
\end{abstract}

Key Words: Connectome, Brain map, Informatics
The deciphering of the entire wiring diagram of neuronal connections will explain how the brain and mind works. While the mapping of physical connectomes at the structural level has been completed in Caenorhabditis elegans, whose nervous systems are composed of 302 neurons and about 7,000 synapses (White et al., 1986), mapping of the human brain, which has approximately 80 billion neurons, is a far more difficult task (Azevedo et al., 2009). Connectomes with cellular resolution are indispensable for evaluating the feasibility of brain modeling. One good example of this is a recent study by Markram et al. (2015), which combined morphological and electrophysiological data within a very small volume of rat cerebral cortex to create virtual neural circuits by digital reconstruction (Markram et al., 2015). This virtual brain circuit allowed for the simulation of neuronal information processes in a given neocortical region. In addition to determining specific cell types and identifying their specific connections, the dynamic nature of neural processing and structural plasticity, a modulation of the exchange of information among neurons, should be incorporated with anatomical connectome data to predict brain function. Integration of such a large amount of data with different modalities is difficult and time-consuming, and the human connectome is in its infancy. Currently, many national and international collaboratory groups have been organized for brain mapping, and the Korean government has also put effort into the Korea Brain Initiative (Jeong et al., 2016).

Many research groups have raised concerns about brain mapping projects (DeFelipe, 2010). The main critiques of the connectome are primarily associated with the technical immaturity/uncertainty of the large-scale collection and processing of the data. Most brain mapping projects are focused on the collection of anatomical and gene expression data. Although these information sets are fundamental

(a) This is an open-access article distributed under the terms of the Creative Commons Attribution Non-Commercial License (http://creativecommons.org/licenses/by-nc/4.0) which permits unrestricted noncommercial use, distribution, and reproduction in any medium, provided the original work is properly cited.

Copyrights @ 2016 by Korean Society of Microscopy 
for the understanding of brain function, if other essential components of brain function are not included (e.g., the dynamic nature of neural wiring), the collected data may not address how the brain works. Furthermore, it is difficult to annotate each circuit with its functional role, because neural circuits are often utilized for different functions in a contextdependent manner. It has also been demonstrated that brain regions exhibit high-level variations among individuals, making standardization difficult. These concerns emphasize the limitations of current anatomy-based mapping strategies, and significant technical improvements are required to complete functional brain mapping.

Currently, most anatomical analyses of brain mapping are based on whole imaging of a defined unit volume. For example, serial block electron microscopy and related methods for microscale connectome analysis focus on detailed structures with a unit volume, and the realignment of this information to cover a larger volume. A major tool for macro-connectomes is magnetic resonance imaging (MRI), and this analysis is based on voxels (three-dimensional volume units). The functional MRI which uses of blood oxygen-level dependent signals or diffusion tensor images for projection mapping all utilize voxel-based analyses, indicating that the data from all major macro-connectomics are composed of unit information. Furthermore, classical electrode based recordings obtain "unit" activity that originates from the collective activities of neurons near the electrodes. These block-based approaches are a simple way to archive data, and thus have merit for large-scale database formulation, and subsequent analyses. The actual connectome is, however, formulated by neurons, and should be interpreted as the specific connections of individual neurons or groups of neuron types at a cellular level. This raises an important question as to how one can identify individual neurons. In $C$. elegans or flies, the number of neurons is relatively small, and currently available techniques allow cellular-level analysis of neural activity (Bargmann, 2012). In these species, a single type of neurons can be labeled or specifically modulated by the use of genetic tools based on the cell-type-specific expression of genes (Chiang et al., 2011). Similarly, cell-type dependent transgenic strategies have been successfully applied to mouse brain mapping (He et al., 2016). Because the number of neurons (or likely neuronal types) in the mouse brain is far larger than the number of cell types that can be

Table 1. Database and resources for brain mapping of many model organisms

\begin{tabular}{|c|c|}
\hline Model species & Resources \\
\hline \multirow[t]{2}{*}{ Caenorhabditis elegans } & http://www.openworm.org \\
\hline & http://www.wormatlas.org/neuronalwiring.html \\
\hline \multirow[t]{4}{*}{ Drosophila } & https://www.janelia.org/project-team/flyem \\
\hline & https://neurokernel.github.io/about.html \\
\hline & http://www.virtualflybrain.org/site/vfb_site/overview.htm \\
\hline & http://flybrain.neurobio.arizona.edu/Flybrain/html/index.html \\
\hline \multirow[t]{2}{*}{ Zebrafish } & http://zebrafishbrain.org \\
\hline & http://vibez.informatik.uni-freiburg.de \\
\hline Xenopus & http://www.xenbase.org/anatomy/atlas.do \\
\hline \multirow[t]{2}{*}{ Chick } & http://avianbrain.org/nomen/Chicken_Atlas.html \\
\hline & http://www.avianbrain.org/atlases.html \\
\hline \multirow[t]{7}{*}{ Mouse and rat } & http://mouse.brain-map.org/static/atlas \\
\hline & http://www.hms.harvard.edu/research/brain/atlas.html \\
\hline & http://www.imaging.org.au/AMBMC/Cortex \\
\hline & http://www.gensat.org/index.html \\
\hline & http://www.mbl.org/mbl_main/atlas.html \\
\hline & http://www.proteinatlas.org/humanproteome/mouse+brain \\
\hline & http://larrywswanson.com/?page_id=164 \\
\hline \multirow[t]{3}{*}{ Primates } & http://www.blueprintnhpatlas.org \\
\hline & https://scalablebrainatlas.incf.org/macaque/DB09 \\
\hline & https://gene-atlas.bminds.brain.riken.jp \\
\hline \multirow[t]{3}{*}{ Human } & http://atlas.brainnetome.org \\
\hline & http://human.brain-map.org \\
\hline & http://www.proteinatlas.org/humanproteome \\
\hline \multirow[t]{2}{*}{ Multi-species } & http://www.brain-map.org https://scalablebrainatlas.incf.org/main/index.php \\
\hline & http://brainmaps.org \\
\hline
\end{tabular}


determined by gene expression (and is actually larger than total number of mouse genes), double or triple combinatorial systems and additional strategies should be introduced to label selective connectivity in the mammalian brain. Other genetic tools for the selective labeling of functional synapses (e.g., mammalian GFP reconstitution across synaptic partners and split horseradish peroxidase) can also be used to explore the micro-level determination of the synaptic connections of two selective neuronal populations.

Brain clearing techniques are increasingly utilized for mesoscale brain mapping. The increased transparency of the whole brain allows for the avoidance of time-consuming processes like the physical sectioning of brain tissue and reconstruction of the information into three dimensions. In particular, hydrogel-based tissue clearing methods have merit for their durability and high transparency. These methods have been successfully applied to address longrange neuronal projections at a cellular level (Chung et al., 2013). The acceleration of the speed of tissue clearing can be achieved with improved electrophoretic tissue chambers and optimization of the tissue processing procedure, which will expedite large-scale brain mapping (Lee et al., 2016a). In addition to hydrogel-based methods, solvent-based tissue clearing methods are also frequently utilized for the observation of relatively small specimens such as mouse spinal cord and embryos (Lee et al., 2016b; Renier et al., 2014). With the improvement of optical imaging tools, tissue-clearingbased methods will become a major workflow for mesoscale whole brain mapping. These cell-based approaches could be combined with block-based approaches using informatics, which will enhance the value of the brain maps.

Many collaborator works focused on brain mapping have tried to collect whole data from different species with different experimental technologies. Now, many brain maps for many different species are available (Table 1). Considering that these model species have both unique and common features in terms of brain structure and function, comparative analysis of multi-species connectomes will provide valuable insights into how the brain works. In this respect, it should be noted that multiple genomes have been compared after the completion of the genome sequencing of many species, to speculate on gene functions and the fundamental role of the genes. For instance, proto-chromosomes, ancestral chromosomes, have been deduced based on phylogenetic comparisons and the evolutionary histories of species in genome research, which allow for the analysis of the natural history of gene functions. Likewise, it might be possible to form proto-connectomes that allow for the comparative evaluation of neural networks. Zador et al. (2012) have proposed an ingenious idea to transform connectomics problems into sequencing problems. Recently, this group released the Multiplexed Analysis of Projections by Sequencing technique, which is a proof-ofconcept of the idea solving connectomes problems with sequencing-based techniques (Kebschull et al., 2016). In brief, they utilized a viral system containing random RNA sequences (barcodes) to infect large numbers of neurons within a given area, which resulted in the filling of each projection axon with different barcode RNAs. Following systematic dissection of the whole brain into small blocks, the barcodes in each block were read by a massive RNA sequencer. In this way, the researchers were able to transform the connectome into sequence information. Similar methods for lineage readout techniques might be combined for the assessment of dynamic alterations in connectomes (Frieda et al., 2016). A unique feature of this strategy is that it is not limited by the resolution and quality of imaging tools. Because sequence-based informatics tools have advanced greatly thanks to the HGP, this transformation may expedite brain mapping projects by removing the need for microscopic imaging tools. This will be just an initial trial of the conversion of anatomy-based brain mapping to information-based mapping.

\section{CONFLICT OF INTEREST}

No potential conflict of interest relevant to this article was reported.

\section{REFERENCES}

Azevedo F A, Carvalho L R, Grinberg L T, Farfel J M, Ferretti R E, Leite R E, Lent R, and Herculano-Houzel S (2009) Equal numbers of neuronal and nonneuronal cells make the human brain an isometrically scaled-up primate brain. J. Comp. Neurol. 513, 532-541.

Bargmann C I (2012) Beyond the connectome: how neuromodulators shape neural circuits. Bioessays 34, 458-465.

Chiang A S, Lin C Y, Chuang C C, Chang H M, Hsieh C H, Yeh C W, Shih C T, Wu J J, Wang G T, and Chen Y C (2011) Three-dimensional reconstruction of brain-wide wiring networks in Drosophila at singlecell resolution. Curr. Biol. 21, 1-11.
Chung K, Wallace J, Kim S Y, Kalyanasundaram S, Andalman A S, Davidson T J, Mirzabekov J J, Zalocusky K A, Mattis J, and Denisin A K (2013) Structural and molecular interrogation of intact biological systems. Nature 497, 332-337.

DeFelipe J (2010) From the connectome to the synaptome: an epic love story. Science 330, 1198-1201.

Frieda K L, Linton J M, Hormoz S, Choi J, Chow K H K, Singer Z S, Budde M W, Elowitz M B, and Cai L (2016) Synthetic recording and in situ readout of lineage information in single cells. Nature doi: 10.1038/ nature20777. 
He M, Tucciarone J, Lee S, Nigro M J, Kim Y, Levine J M, Kelly S M, Krugikov I, Wu P, and Chen Y (2016) Strategies and tools for combinatorial targeting of GABAergic neurons in mouse cerebral cortex. Neuron 91, 1228-1243.

Jeong S J, Lee H, Hur E M, Choe Y, Koo J W, Rah J C, Lee K J, Lim H H, Sun W, and Moon C (2016) Korea brain initiative: integration and control of brain functions. Neuron 92, 607-611.

Kebschull J M, da Silva P G, Reid A P, Peikon I D, Albeanu D F, and Zador A M (2016) High-throughput mapping of single neuron projections by sequencing of barcoded RNA. BioRxiv 10, 054312.

Lee E, Choi J, Jo Y, Kim J Y, Jang Y J, Lee H M, Kim S Y, Lee H J, Cho K, and Jung N (2016a) ACT-PRESTO: rapid and consistent tissue clearing and labeling method for 3-dimensional (3D) imaging. Sci. Rep. 6. 18631
Lee E, Kim H J, and Sun W (2016b) See-through technology for biological tissue: 3-dimensional visualization of macromolecules. Int. Neurourol. J. 20, S15.

Markram H, Muller E, Ramaswamy S, Reimann M W, Abdellah M, Sanchez C A, Ailamaki A, Alonso-Nanclares L, Antille N, and Arsever $S$ (2015) Reconstruction and simulation of neocortical microcircuitry. Cell 163, 456-492.

Renier N, Wu Z, Simon D J, Yang J, Ariel P, and Tessier-Lavigne M (2014) iDISCO: a simple, rapid method to immunolabel large tissue samples for volume imaging. Cell 159, 896-910.

White J G, Southgate E, Thomson J N, and Brenner S (1986) The structure of the nervous system of the nematode Caenorhabditis elegans. Philos. Trans. R. Soc. Lond. B. Biol. Sci. 314, 1-340.

Zador A M, Dubnau J, Oyibo H K, Zhan H, Cao G, and Peikon I D (2012) Sequencing the connectome. PLOS Biol. 10, e1001411. 